\title{
Modification of the Coulomb's Law in an Optical Near-Field Atomic Dipole Model
}

\author{
Dmytro Vasylenko*, Petro Kravchuk, Valerii Grygoruk \\ Faculty of Radio Physics, Electronics and Computer Systems, Taras Shevchenko National University of Kyiv, Kyiv, Ukraine \\ Email address: \\ vda7@ukr.net(D. Vasylenko) \\ ${ }^{*}$ Corresponding author \\ To cite this article: \\ Dmytro Vasylenko, Petro Kravchuk, Valerii Grygoruk. Modification of the Coulomb's Law in an Optical Near-Field Atomic Dipole Model. \\ American Journal of Optics and Photonics. Vol. 6, No. 2, 2018, pp. 20-24. doi: 10.11648/j.ajop.20180602.11
}

Received: September 4, 2018; Accepted: September 25, 2018; Published: October 26, 2018

\begin{abstract}
In this paper, a model of a dipole with an atomic structure was considered, instead of the standard dipole model with point unlike charges and the Hertzian dipole model, which have significant drawbacks. It is shown that in the atomic dipole the Coulomb's law in the classical formulation does not work. Therefore, the Coulomb's law needs to be modified. A formula is proposed for the force of the dipole that arises between unlike charges in the process of dipole oscillations and the decompensation/compensation of their fields. The representation of the dependence of the interaction force between unlike charges on the distance between them was shown for three zones: the oscillation zone in which the proposed dipole force formula works, the ionization zone with electron shell detachment from the nucleus and coverage zone of the Coulomb's law between the divided charges formed as a result of ionization of the atom. The dynamics of the process of oscillation of the atomic dipole in four phases (quarters of the period) is investigated. It is shown that the reactive energy flows first emerge from the dipole, and then return to it, while the active energy flows always propagate from the dipole to the far zone. The mechanism of wave propagation of the radiation field is shown.
\end{abstract}

Keywords: Coulomb's Law, Intra-Dipole Vectors, Compensation, Lines of Force

\section{Introduction}

There is a model of the Hertzian dipole in classical electrodynamics, which is widely used even today [1-5]. The Hertzian dipole is an infinitesimal structureless element of the current - in fact a «black box». There are no real intra-dipole vectors $\mathrm{E}, \mathrm{H}$, and $\mathrm{S}$ in the Hertzian model, which does not allow us to consider the physical mechanism of formation of reactive and active energy fluxes of the optical near field and the radiation field, respectively. The Hertzian dipole has no concept of the intra-charge force $\mathrm{F}$ and, consequently, the equilibrium mechanism of the steady-state oscillation amplitude $l_{0}$ of the dipole charges from the value of the external inducing field $\mathrm{E}_{0}$.

Recently, more and more studies, both theoretical [6-9], and experimental [10-13], show a fundamental difference between physical processes occurring in optical near and far fields. The optical near field of the Hertzian dipole was investigated in papers $[14,15]$. As a rule, a dipole is a system consisting of two point charges $+q$ and $-q$ located at a distance $l_{0}$ from each other, and $r \gg l_{0}$ (where $r$ is the distance from the dipole to the observation point) [16]. The presence of point charges of this standard model should allow the use of the Coulomb's law $F \sim\left(q_{1} \cdot q_{2}\right) / l^{2}$. But the attempt to apply Coulomb's law to these charges leads to a «Coulomb's collapse» at the zero point (where charges coincide) as $l \rightarrow 0$, in which the dipole transforms into a «Coulomb's black hole», since $F \rightarrow \infty$. And no external field (with finite force) can detach charges and polarize the atom.

\section{Modification of Coulomb's Law}

It is known that when an external field is applied, the atoms are polarized and their dipole moments oscillate with the participation of intra-charge forces. But this is not the force of the Coulomb's law - inside the atomic dipole Coulomb's law does not work. Obviously, a new dipole model and another formula for the intra-dipole force between the charges of the oscillating dipole are required. It is necessary to 
fundamentally change the approach to understanding the processes occurring during dipole oscillations.

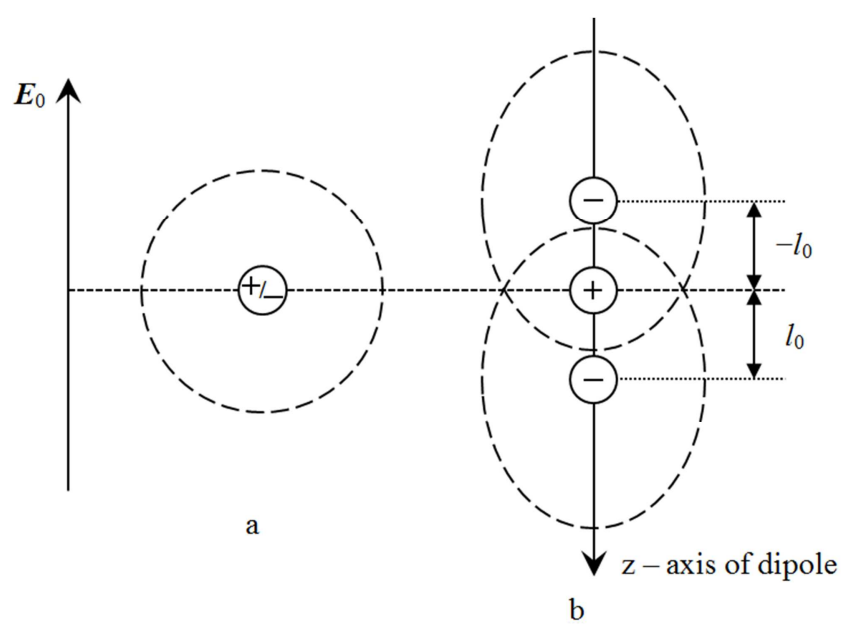

Figure 1. Polarization of an atom in an external electric field ( $a$ - atom is unpolarized, $b$-atom is polarized and oscillates). "+» is the positive charge of the atomic nucleus, "-» is the equivalent negative charge of the electron cloud. The dotted line indicates an electron cloud.

In contrast to the structureless model of the Hertzian dipole or the standard model with point charges, it was suggested to use for the dipole model the real charge structure of the atom in which the positive charge of the nucleus is always inside the electron shell and called it the «atomic dipole model». This model functions for any atoms and molecules, since all of them are polarized in an external field, forming oscillating dipoles (Figure 1).

Since the electron orbitals are centrally symmetric, instead of the electron charges spatially distributed over them, the concept of «equivalent negative charge» $(-q)$ was introduced into the proposed dipole model equal to the sum of the charges of all electrons of the atom and its electronic orbitals located at the center of symmetry. This is not an actual charge, but its equivalent model, which facilitates the description of the physical mechanisms that occur when oscillating a real dipole with an atomic structure.

The center of the positive charge $+q$ (nucleus) is always inside the electron shell, so the charge fields $+q$ and $-q$ always overlap and partially compensate for the other. Without an

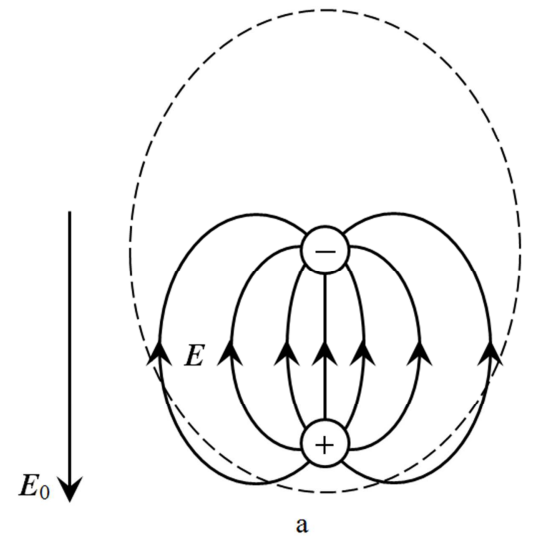

external field $\left(E_{0}=0\right)$, the centers of charges $+q$ and $-q$ are combined, the compensation of the charge fields is complete and the atom is unpolarized (Figure 1, a).

The dipole oscillates in an external field $\left(E_{0} \neq 0\right)$ (Figure 1, b). The interference of the fields of two charges, which are identical in magnitude but opposite in sign, occurs in the process of oscillation. The nucleus is orders of magnitude more massive than electrons and therefore practically motionless. Centers of unlike charges then move away from each other (diverge), then converge. In doing so, they compensate for the smaller, then the greater part of the quasi-static fields of each other and their lines of force.

The fields themselves remain at their charges, but the interference of fields of unlike charges reduces their external force manifestation, changing the density of the lines of force. A significant part of the lines of force of both charges is in a bound state of mutual compensation and does not manifest itself in force interaction.

This can be called the interference of quasistatic fields. Thus, the near field is the intrinsic quasistatic fields (lines of force) of Coulomb and Biot-Savart of two oscillating dipole charges whose fields interfere with its oscillation. But outwardly it looks like a pulsation of the electromagnetic field, reactive in phase shifts and alternating energy flows.

It is important to note that the electron shell always contains within itself not only an equivalent negative charge, but also a positive charge of the nucleus, that is, in fact the entire dipole. In this model, the axis of the dipole is a straight line passing through the center of the positive charge of the nucleus and the center of the equivalent negative charge of the electron cloud of the atom.

When an external field is applied, the electron shell is displaced (stretched) relative to the nucleus against the field and the charge centers $+q$ and $-q$ diverge (Figure 2, a). The atom is polarized due to a partial decompensation of the overlapping fields of divergent unlike charges.

When the sign of the external field changes, the direction of the displacement of the electron shell changes to the opposite direction (Figure 2, b; 1, b). The lines of force of the electric field of a dipole, according to electrostatics, begin with a positive charge and end in a negative charge.

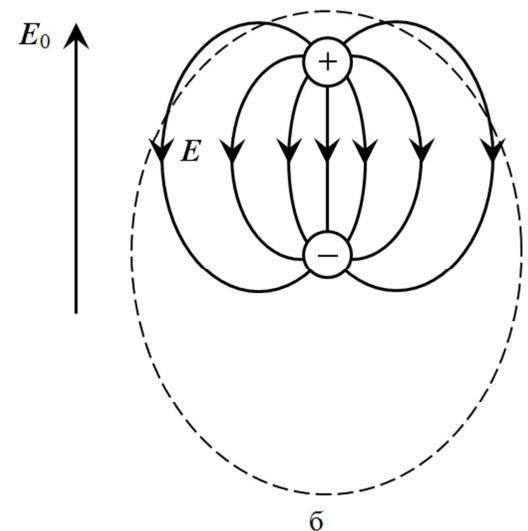

Figure 2. Atomic dipole in an external electric field for different directions of the $E_{0}$ vector (a and b). "+" Is the positive charge of the atomic nucleus, "-" is the equivalent negative charge of the electron cloud. The dotted line indicates an electronic cloud. 
They are always opposite to the external field $E_{0}$ and therefore partially compensate for it, which explains the decrease in the amplitude of the wave in the dielectric by a factor of $\varepsilon$ (where $\varepsilon$ is the permittivity).

In the process of displacement of the equivalent negative charge of the electron shell, a current arises that, according to the Biot-Savart law, generates a closed field line of the toroidal quasistatic magnetic field around the streamline (the axis of the dipole $l$ ). The direction of the current is opposite to the displacement of the electron shell, that is, it coincides with the direction of the external field.

An important element of the proposed atomic model is that in the process of polarization (with the change in the dipole $\operatorname{arm} l$ ), the magnitude of the decompensation of the fields varies (this is not present in the standard model and the Hertzian dipole model). The compensation of the fields is constant in the standard model of the dipole, which is untrue. And the Hertzian dipole is structureless, which does not allow us to consider the phenomenon of decompensation in principle.

With increasing external field, the center of the electron shell moves further and further from the positive nucleus, which leads to an even greater decompensation of the overlapping charge fields $+q$ and $-q$. Thus, when the external field changes, the dipole moment $p=q l$ changes due to the effect of the decompensation.

Obviously, to describe the attractive forces between unlike charges in the process of partial decompensation of their fields under the action of an external polarizing field, instead of the Coulomb's law formula, another formula is needed that takes into account the change in the magnitude of the decompensation of the charge fields with the change in the
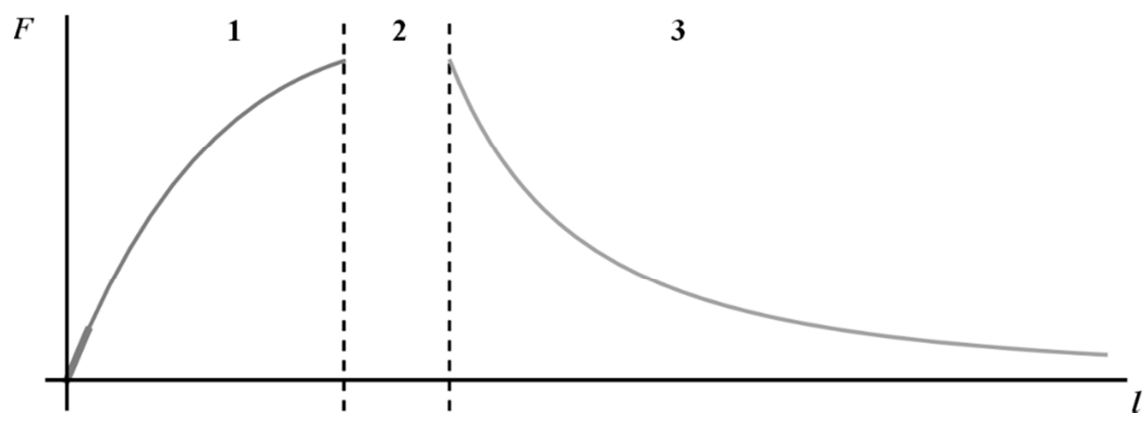

Figure 4. A qualitative representation of the dependence of the interaction force between the charges $+q$ and $-q$ on the distance between them (1 - the oscillation zone in which the dipole force formula (1) works, 2 - the ionization zone with electron shell detachment from the nucleus, 3 - the zone of action of the Coulomb's law between the divided charges formed as a result of ionization of the atom).

If the external field $E_{0}$ dissociates charges then, according to the dipole force formula, they are on the contrary - attracted to each other. So the force action of the external field $E_{0}$ and the dipole force $\mathrm{F}$ are directly opposite and the further growth of the amplitude $l_{0}$ of the oscillations of the dipole stops when the equilibrium of these forces is reached.

Figure 4 shows the graphs of the dependence of the interaction force between unlike charges on the distance between them.

Oscillation of the atom always occurs at the beginning of the first zone (the bold portion of the line) prior to its dipole arm $l$ (Figure 3).

A functional formula in the atomic dipole model for the dipole force that arises between unlike charges in the process of decompensating their fields was proposed:

$$
E(l) \sim E-E_{c}(l)
$$

where $E$ is the charge field strength; $E_{c}(l)$ is the intensity of the compensated part of the charge field (compensation).

Wherein $l \uparrow \Rightarrow E_{c} \downarrow \Rightarrow F \uparrow\left(l \downarrow \Rightarrow E_{c} \uparrow \Rightarrow F \downarrow\right)$. Also, the formula for the dipole force can be represented in the following form:

$$
E(l) \sim E_{d c}(l),
$$

where $E_{d c}(l)=E-E_{c}(l)$ is the intensity of the uncompensated part of the charge field (decompensation).

Here $l \uparrow \Rightarrow E_{d c} \uparrow \Rightarrow F \uparrow\left(l \downarrow \Rightarrow E_{d c} \downarrow \Rightarrow F \downarrow\right)$. There is no problem of «Coulomb's collapse» at the zero point in this formula, since:

$$
l \rightarrow 0 \Rightarrow E_{d c} \rightarrow 0 \Rightarrow F \rightarrow 0 .
$$

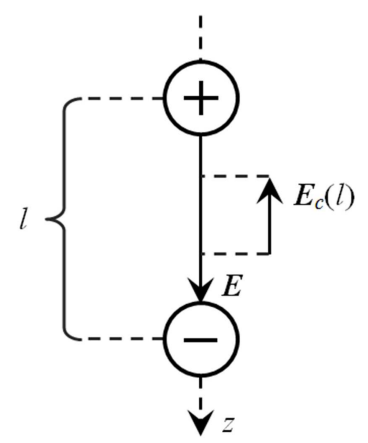

Figure 3. The phenomenon of decompensation (and compensation) of the Figure 3. The phenoment
fields of dipole charges.

3

ionization. The nucleus remains inside the electron shell and the decompensation of fields of unlike charges in an external field is partial. With larger external fields, nonlinearity (a thin portion of the line) begins to appear, and with further growth, ionization of the atom occurs (the second zone), which means the electron shell detaches from the nucleus. In the third zone, the electron shell and the nucleus are already detached in space from each other. And in this zone Coulomb's law is already working.

In this structural atomic model, there are intra-dipole quasistatic vectors $\mathrm{E}, \mathrm{H}$ and $\mathrm{S}$, which makes it possible to 
consider the mechanisms of the formation of energy fluxes in the oscillation process: as reactive $\mathrm{S}_{r}=\mathrm{E} \times \mathrm{H}$ (where $\mathrm{E}$ and $\mathrm{H}$ are the components of electric and magnetic near-field strengths that have phase shift $\Delta \varphi=\pi / 2$ ) and active $\mathrm{S}_{a}=\mathrm{E} \times \mathrm{H}$ (where $\mathrm{E}$ and $\mathrm{H}$ are the components of the electric and magnetic strengths of the active radiation field with phase shift $\Delta \varphi=0$ ). At the same time, as it became known, $\mathrm{E} \equiv \mathrm{E}_{d c}$ and, accordingly, $\mathrm{H} \equiv$ $\mathrm{H}_{d c}$ (where $\mathrm{H}_{d c}$ is the intensity of the uncompensated part of the magnetic field of the moving charge).

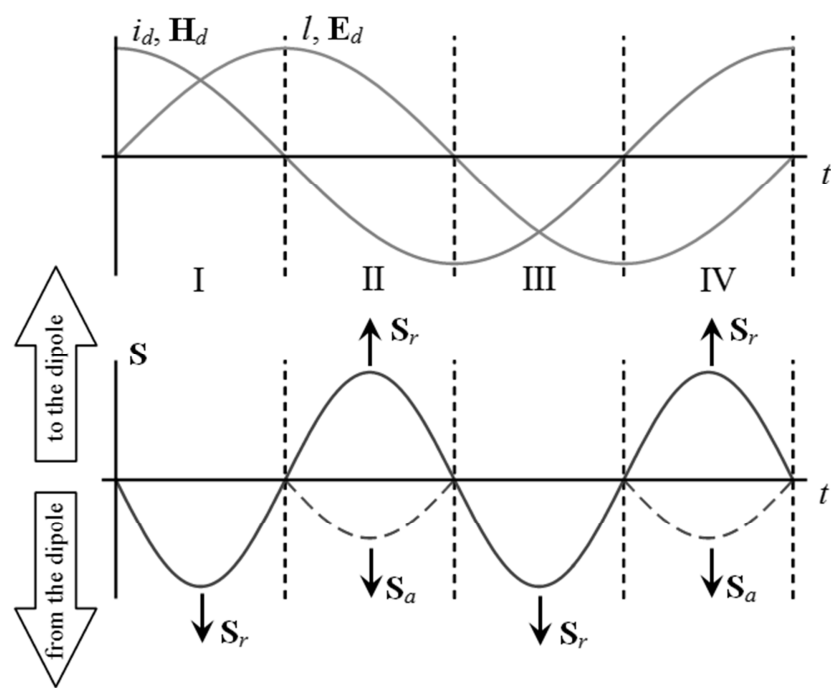

Figure 5. The correspondence between the values of $i_{d}, H_{d}$, as well as of $E_{d}$ as functions of the dipole arm $l$, and the directions of reactive $S_{r}$ and active $S_{a}$ energy flows in 4 phases of the oscillation period.

So, the near field is a field of decompensation. It appears and intensifies when unlike charges in the external inducing field diverge. And it disappears when they pass through the zero point, that is, they are combined and completely compensate for the interference of each other's fields with interference (the intrinsic fields are always inseparable from their charges). When the charges disagree, the full compensation is violated and the uncompensated near field begins to increase. When converging, on the contrary, the interfering fields of unlike charges are combined up to full compensation of external fields when they coincide at the zero point. Thus, the fields of both charges are an "interference

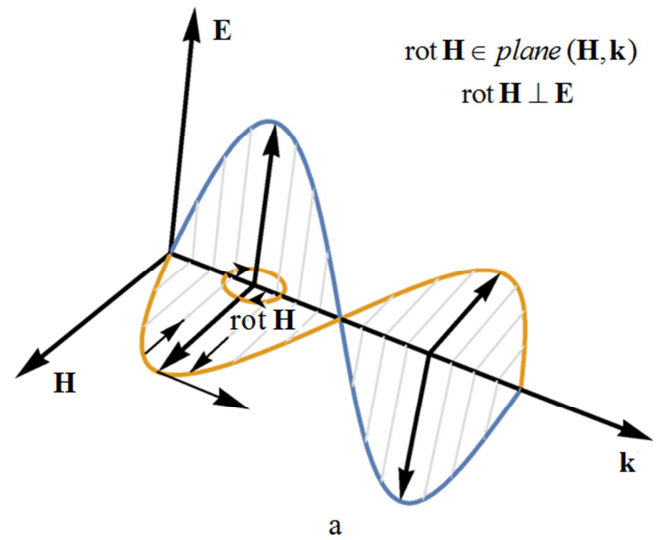

mask" for each other, connecting a part of the lines of force that is changing during the oscillation process.

Figure 5 shows the dynamics of the process of oscillation of an atomic dipole. As can be seen, the oscillation consists of 4 phases (quarters of the period). According to this figure, it is possible to trace the correspondence between the current $i_{d}$, the magnetic field $\mathrm{H}_{d}$, and also $\mathrm{E}_{d}$ as functions of the distance $l$ between unlike dipole charges, and the directions of the reactive $\mathrm{S}_{r}$ and active $\mathrm{S}_{a}$ energy flows in 4 phases of the oscillation period.

\section{Wave of Radiation Field}

Below are two Maxwell equations (Ampere's circuital law and Faraday's law of induction) for vacuum:

$$
\begin{aligned}
\operatorname{rot} \boldsymbol{H} & =\boldsymbol{j}+\varepsilon_{0} \frac{\partial \boldsymbol{E}}{\partial t}, \\
\operatorname{rot} \boldsymbol{E} & =-\mu_{0} \frac{\partial \boldsymbol{H}}{\partial t}
\end{aligned}
$$

To find out the mechanism of electromagnetic wave propagation, the initial point was chosen so that the values of $\mathrm{E}$ and $\mathrm{H}$ were maximum (Figure 6). This determines the behavior of the E vector at the next instant of time - its modulus can only decrease.

A decrease in E, according to (4), will cause rot $\mathrm{H}$. The vortex of the magnetic field at this point lies in the $(\mathrm{H}, \mathrm{k})$ plane (Figure 6, a). The magnetic field of the vortex is summed with the value of $\mathrm{H}$ of the wavefront and subtracted from the backwave front. Consequently, the maximum of $\mathrm{H}$ is displaced forward (in the direction of $\mathrm{k}$ ), and, therefore, at the initial point, $\mathrm{H}$ decreases.

In accordance with (5), a decrease in $\mathrm{H}$ at the initial point will cause in the orthogonal plane $(\mathrm{E}, \mathrm{k})$ a rot $\mathrm{E}$ (Figure $6, \mathrm{~b}$ ), the value of which will be added to the wavefront $\mathrm{E}$ and subtracted from the backwave front. The maximum of $\mathrm{E}$ will move forward (in the direction of $\mathrm{k}$ ), and at the initial point $\mathrm{E}$ decreases. This again will cause such a rot $\mathrm{H}$, which again will move the maximum $\mathrm{H}$ forward, and so on.

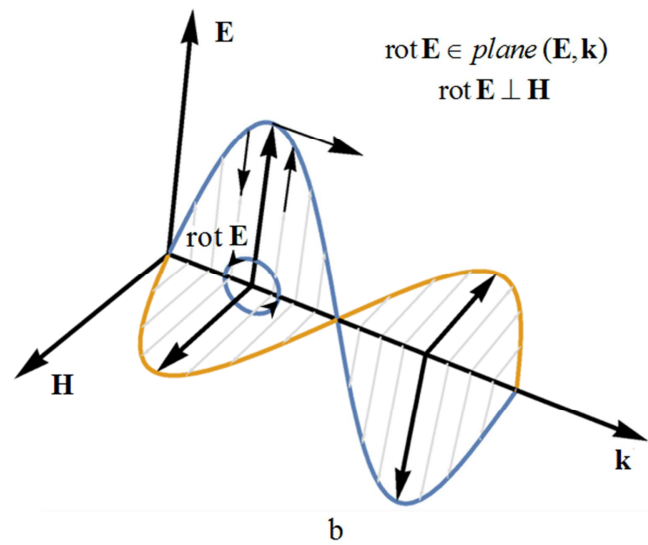

Figure 6. Vector representation of the active electromagnetic wave of the radiation field with the image of the rot $H$ (a) and the rot $E$ (b). The amplitudes $E$ and $H$ are in phase $(\Delta \varphi=0)$. 
Thus, the entire wave by moving its crests will spread forward (in the direction of $\mathrm{k}$ ).

The wave is not propagating oscillations, as erroneously it is sometimes said. Any electromagnetic field can be decomposed into active and reactive components. In oscillations $\Delta \varphi=\pi / 2$ and therefore when the amplitude of one of the fields is maximal, the amplitude of the second is zero and vice versa. And in the waves $\Delta \varphi=0$ - the amplitudes are in phase.

Hence, the energies of the reactive fields are completely pumped into each other in the entire region of oscillations. This does not happen in waves in which the amplitudes $\mathrm{E}$ and $\mathrm{H}$ are never zeroed - they simply move the ridges of each other in the propagation direction $(\mathrm{k})$.

\section{Conclusions}

A dipole model with an atomic structure was proposed and it was called the «atomic dipole model», which is closer to reality than the Hertzian dipole model or the classical model with point charges. It is shown that when the electron shell oscillates under the action of an external field, a partial decompensation of the charge fields occurs, which depends on the dipole arm 1 and determines the attractive force of the charges of different charges.

It is shown that in the atomic dipole the Coulomb's law in the classical formulation does not work. Therefore, the Coulomb's law needs to be modified. A formula is proposed for the force of the dipole that arises between unlike charges in the process of dipole oscillations and the decompensation / compensation of their fields.

The representation of the dependence of the interaction force between unlike charges on the distance between them was shown for three zones: the oscillation zone in which the proposed dipole force formula works, the ionization zone with electron shell detachment from the nucleus and coverage zone of the Coulomb's law between the divided charges formed as a result of ionization of the atom.

The dynamics of the process of oscillation of the atomic dipole in four phases (quarters of the period) is investigated. It is shown that the reactive energy flows first emerge from the dipole, and then return to it, while the active energy flows always propagate from the dipole to the far zone.

The mechanism of wave propagation of the radiation field is shown.

\section{References}

[1] K. Sainath, F. L. Teixeira, (2014). Spectral-Domain-Based Scattering Analysis of Fields Radiated by Distributed Sources in Planar-Stratified Environments with Arbitrarily Anisotropic Layers. Physical Review E, 90 (6), 1-17.

[2] H. Moon, F. L. Teixeira, B. Donderici, (2014). Stable pseudoanalytical computation of electromagnetic fields from arbitrarily-oriented dipoles in cylindrically stratified media. Journal of Computational Physics, 273, 118-142.

[3] Ch. Christakis, K. Ioannidi, S. Sautbekov, P. Frangos, S. K. Atanov, (2014). The radiation problem from a vertical short dipole antenna above flat and lossy ground. Novel formulation in the spectral domain with closed form analytical solution in the high frequency regime. Elektronika ir Elektrotechnika, 20 (9), 35-38.

[4] Sutinjo, J. O'Sullivan, E. Lenc, R. B. Wayth, S. Padhi, P. Hall, S. J. Tingay, (2015). Understanding instrumental Stokes leakage in Murchison Widefield Array polarimetry. Radio Science, 50 (1), 52-65.

[5] M. Arminjon, (2017). Charge conservation in a gravitational field in the scalar ether theory. Open Physics, 15, 877-890.

[6] Y. M. Morozov and A. S. Lapchuk, (2016). Signal of microstrip scanning near-field optical microscope in far- and near-field zones. Applied Optics, 55 (13), 3468-3477.

[7] H. M. K. Wong, M. K. Dezfouli, S. Axelrod, S. Hughes and A. S. Helmy, (2017). Theory of hyperbolic stratified nanostructures for surface-enhanced Raman scattering. Physical Review B, 96, 205112.

[8] K. Staliunas, P. Markoš, V. Kuzmiak, (2017). Scattering properties of a PT dipole. Physical Review A, 96 (4), 043852.

[9] J. Yuffa, Y. Gutierrez, J. M. Sanz, R. A. de la Osa, J. M. Saiz, F. González, F. Moreno and G. Videen, (2016). Near- and far-field scattering resonance frequency shift in dielectric and perfect electric conducting cylinders. Journal of the Optical Society of America A, 33 (3), 391-395.

[10] K. Kobayashi, T. Kawazoe, and M. Ohtsu, (2005). Importance of multiple-phonon interactions in molecular dissociation and nanofabrication using optical near fields. IEEE Transactions on Nanotechnology, 4 (5), 517-522.

[11] E. Tucker, J. D'Archangel and G. Boreman, (2017). Near- and far-field investigation of dark and bright higher order resonances in square loop elements at mid-infrared wavelengths. Optics Express, 25 (5), 5594-5608.

[12] D. Cao, A. Cazé, M. Calabrese, R. Pierrat, N. Bardou, S. Collin, R. Carminati, V. Krachmalnicoff and Y. De Wilde, (2015). Mapping the Radiative and the Apparent Nonradiative Local Density of States in the Near Field of a Metallic Nanoantenna. ACS Photonics, 2 (2), 189-193.

[13] R. C. Boutelle, D. Neuhauser, S. Weiss, (2016). Far-Field Super-resolution Detection of Plasmonic Near-Fields. ACS Nano, 10 (8), 7955-7962.

[14] V. S. Sydorenko, Yu. O. Gayday, S. V. Zhyla, (2005). Features of the near field of the Hertz Dipole. Bulletin of the University of Kyiv. Series: Physics \& Mathematics, 2, 365-372.

[15] V. S. Sydorenko, Yu. O. Gayday, S. V. Zhyla, O. V. Sinkevych, (2003). Distribution of the Poynting vector in the near field of the Hertz dipole. Bulletin of National Taras Shevchenko University of Kyiv. Radio Physics and Electronics, 1, 55-59.

[16] L. D. Landau, E. M. Lifshits, Theoretical Physics, vol. 2: The Classical Theory of Fields. Moscow: Nauka, 1973, p. 504. 